\title{
Multiplicity of nontrivial solutions for a class of nonlinear Kirchhoff-type equations
}

Hongliang Liu', Haibo Chen ${ }^{1 *}$ and Yueding Yuan ${ }^{2}$

\section{"Correspondence:}

math_chb@csu.edu.cn

${ }^{1}$ School of Mathematics and

Statistics, Central South University,

Changsha, Hunan 410083, P.R. China

Full list of author information is

available at the end of the article

\begin{abstract}
This paper deals with the existence of nontrivial solutions of the following Kirchhoff-type equation: $-\left(a+b \int_{\mathbb{R}^{N}}|\nabla u|^{2} d x\right) \Delta u+V(x) u=f(x, u)$, in $\mathbb{R}^{N}$. Under more relaxed assumptions on $V$ and $f$, some new criteria on the multiplicity of nontrivial solutions are established by combining Morse theory with local linking and Clark's theorem.
\end{abstract}

MSC: $58 \mathrm{E} 05 ; 35 \mathrm{~J} 20 ; 35 \mathrm{~B} 38$

Keywords: Morse theory; local linking; critical group; Kirchhoff-type equation

\section{Introduction and main results}

In this paper, we investigate the multiplicity of nontrivial solutions to the following Kirchhoff-type equation:

$$
-\left(a+b \int_{\mathbb{R}^{N}}|\nabla u|^{2} d x\right) \Delta u+V(x) u=f(x, u), \quad \text { in } \mathbb{R}^{N},
$$

where $N \geq 2, a>0, b \geq 0$ are constants and the potential $V(x)$ satisfies the following condition:

(V) $V(x) \in C\left(\mathbb{R}^{N}, \mathbb{R}\right)$ satisfies $\inf _{\mathbb{R}^{N}} V(x) \geq \alpha>0$.

Problem (1.1) is related to the stationary analog of the equation

$$
u_{t t}-\left(a+b \int_{\Omega}|\nabla u|^{2}\right) \Delta u=h(x, u)
$$

which was presented by Kirchhoff [1] to describe the transversal oscillations of a stretched string, where $u$ denotes the displacement, $h$ is the external force, $b$ represents the initial tension, and $a$ is related to the intrinsic properties of the string. Moreover, a nonlocal effect can also be applied in many biological systems. A parabolic version of problem (1.2) can be used to describe the growth and movement of a particular species. For more details of the background of problem (1.1), we refer the reader to [2,3] and references therein.

In recent years, with the aid of critical point theory and variational methods, problem (1.1) have been widely studied by many authors since Lions [4] proposed an abstract framework. More precisely, Wu [5] studied the existence of nontrivial solution and infinitely

(c) 2015 Liu et al. This article is distributed under the terms of the Creative Commons Attribution 4.0 International License (http://creativecommons.org/licenses/by/4.0/), which permits unrestricted use, distribution, and reproduction in any medium, provided you give appropriate credit to the original author(s) and the source, provide a link to the Creative Commons license, and indicate if changes were made. 
many high energy solutions of problem (1.1) by using a symmetric mountain pass theorem. In [6], Sun and Wu investigated the existence and the non-existence of nontrivial solution of problem (1.1) by using variational methods and explored the concentration of solution. Liu and He [7] discussed the existence of infinitely many high energy solutions of problem (1.1) under superlinear case by variant version of fountain theorem. When $N=3$, Li and Ye considered problem (1.1) with pure power nonlinearities $f(x, u)=|u|^{p-1} u$ in $\mathbb{R}^{3}$. By using a monotonicity trick and a new version of global compactness lemma, they verified that the problem has a positive ground state solution that the result can be viewed as a partial extension of [8] where the authors studied the existence and concentration behavior of positive solutions of problem (1.1). In addition, other interesting results on the related Kirchhoff-type equations can be found in [9-19] and references therein.

However, in a large number of the above works, the authors considered the corresponding problem for the superlinear case. Compared to the superlinear case, there are few papers concerning the case where $f$ is sublinear as $|x| \rightarrow \infty$. For example, the authors in [20] studied problem (1.1) with the sublinear case. By the genus properties in critical point theory, they showed that the problem has infinitely many nontrivial solutions.

Motivated by these facts, in this paper, we will study the multiplicity of nontrivial solutions to problem (1.1) with the sublinear growth. But the main tools we use in the present paper are the Morse theory and Clark's theorem, different from [20].

Now, we are ready to state the main results of this paper.

Theorem 1.1 Assume that condition (V) holds and let the following conditions be satisfied.

(f1) $f \in C\left(\mathbb{R}^{N} \times \mathbb{R}, \mathbb{R}\right)$ and there exist constants $1<\delta_{1}<\delta_{2}<\cdots<\delta_{m}<2$, functions $a_{i} \in$ $L^{\frac{2}{2-\delta_{i}}}\left(\mathbb{R}^{N}, \mathbb{R}_{+}\right)(i=1,2, \ldots, m)$ such that

$$
|f(x, u)| \leq \sum_{i=1}^{m} \delta_{i} a_{i}(x)|u|^{\delta_{i}-1}
$$

(f2) There exist $r_{1}>r_{0}>0, \kappa \in(1,2), 2<\vartheta \leq 2^{*}$, and $c_{2}>c_{1}>0$ such that

$$
c_{1}|u|^{\kappa} \leq F(x, u) \leq c_{2}|u|^{\vartheta}, \quad \forall x \in \mathbb{R}^{N}, r_{0}<|u|<r_{1}
$$

where $F(x, u)=\int_{0}^{u} f(x, s) d s$.

Then problem (1.1) possesses at least two nontrivial solutions.

Theorem 1.2 Assume that all the conditions in Theorem 1.1 are satisfied. In addition, the following condition holds.

(f3) $f(x,-u)=-f(x, u), \forall(x, u) \in \mathbb{R}^{N} \times \mathbb{R}$.

Then problem (1.1) possesses at least $k\left(\operatorname{dim} W_{1}=k<+\infty\right.$, where $\left.W_{1} \subset E\right)$ distinct pairs of nontrivial solutions.

It is easy to see that (f2) is still satisfied if the following condition, (f2'), holds. 
(f2') there exist $r_{1}>r_{0}>0, \kappa \in(1,2), 2<\vartheta \leq 2^{*}$, and $c_{2}>c_{1}>0$ such that

$$
c_{1}|u|^{\kappa} \leq u f(x, u) \leq c_{2}|u|^{\vartheta}, \quad \forall x \in \mathbb{R}^{N}, r_{0}<|u|<r_{1} .
$$

Therefore, a direct result of Theorems 1.1 and Theorem 1.2 is the following corollary.

Corollary 1.1 Consider Theorem 1.1 and Theorem 1.2. If the assumption (f2) is replaced by (f2'), then the conclusions still hold.

Remark 1.1 There are some functions satisfying the conditions (f1)-(f3). For example, let $V(x)=5+3 \sin |x|^{2}$ and set $\alpha=1$. Then condition (V) is satisfied. For $\forall(x, u) \in \mathbb{R}^{N} \times \mathbb{R}$, let

$$
f(x, u)=\frac{1+\sin ^{2}|x|}{1+|x|^{\frac{3}{2}}}|u|^{\frac{-4}{5}} u+\frac{1}{e^{|x|}}|u|^{\frac{-2}{3}} u+\frac{3 \cos |x|}{2 e^{3}|x|}|u|^{\frac{-3}{4}} u .
$$

Clearly,

$$
|f(x, u)| \leq \frac{2}{1+|x|^{\frac{3}{2}}}|u|^{\frac{1}{5}}+\frac{1}{e^{|x|}}|u|^{\frac{1}{3}}+\frac{3}{2 e^{3}|x|}|u|^{\frac{1}{4}}
$$

and

$$
F(x, u)=\frac{1+\sin ^{2}|x|}{1+|x|^{\frac{3}{2}}} \frac{5}{6}|u|^{\frac{6}{5}}+\frac{3}{4 e^{|x|}}|u|^{\frac{4}{3}}+\frac{6 \cos |x|}{5 e^{3}|x|}|u|^{\frac{5}{4}} .
$$

Take $2<\vartheta \leq 2^{*}, r_{1}>0,0<\beta<r_{1}^{\frac{4-3 \vartheta}{3}}$, and set

$$
c_{1}:=\beta b_{2}, \quad c_{2}:=3 \max \left\{b_{1} r_{1}^{\frac{6-5 \vartheta}{5}}, b_{2} r_{1}^{\frac{4-3 \vartheta}{3}}, b_{3} r_{1}^{\frac{5-4 \vartheta}{4}}\right\} .
$$

Obviously, $0<c_{1}<c_{2}$ and $c_{1}|u|^{\frac{4}{3}} \leq F(x, u) \leq c_{2}|u|^{\vartheta}$. Thus (f1), (f2), and (f3) are satisfied with $\delta_{1}=\frac{6}{5}, \delta_{2}=\frac{4}{3}, \delta_{3}=\frac{6}{5}, a_{1}=\frac{5}{3\left(1+|x|^{\frac{3}{2}}\right)}, a_{2}=\frac{3}{4 e^{|x|}}, a_{3}=\frac{6}{5 e^{3}|x|}, b_{1}=\frac{1+\sin ^{2}|x|}{1+|x|^{\frac{3}{2}}} \frac{5}{6}, b_{2}=\frac{3}{4 e^{|x|}}$, $b_{3}=\frac{6 \cos |x|}{5 e^{3}|x|}, \kappa=\frac{4}{3}$.

Here, we give the sketch of the proof. First of all, by virtue of the local linking method $[21,22]$, we see that the critical group of functional $J$ at 0 is not isomorphism with 0 , that is, $C_{q}(J, 0) \nsucceq 0$. This means that 0 is a homological nontrivial critical point of functional $J$. And then, using the three point theorem [23, 24], we prove that problem (1.1) has at least two nontrivial solutions (Theorem 1.1). Further, on the basis of Theorem 1.1, we prove that problem (1.1) has $k(1 \leq k<\infty)$ distinct pairs solutions by Clark's theorem (Theorem 1.2) when functional $J$ is even.

The sequel of this paper is organized as follows. Some preliminaries are presented in Section 2 while the proofs of our main results are delegated in Section 3.

\section{Preliminaries}

Throughout this paper, $C$ and $C_{i}$ denote positive constants, which may vary from line to line, $\rightarrow(\rightarrow)$ denotes strong (weak) convergence, $B_{R}$ denotes the ball of radius $R$ and $E^{*}$ denotes the dual space of $E$. 
We will work in the following Hilbert space:

$$
E:=\left\{u \in H^{1}\left(\mathbb{R}^{N}\right): \int_{\mathbb{R}^{N}}\left[|\nabla u|^{2}+V(x)|u|^{2}\right] d x<+\infty\right\}
$$

which is equipped with the inner product

$$
\langle u, v\rangle=\int_{\mathbb{R}^{N}}[\nabla u \nabla v+V(x) u v] d x, \quad u, v \in E,
$$

and the associated norm

$$
\|u\|=\left(\int_{\mathbb{R}^{N}}\left[|\nabla u|^{2}+V(x) u^{2}\right] d x\right)^{\frac{1}{2}}, \quad u \in E
$$

As usual, for $1 \leq p<+\infty$, let

$$
\|u\|_{p}=\left(\int_{\mathbb{R}^{N}}|u|^{p} d x\right)^{\frac{1}{p}}, \quad u \in L^{p}\left(\mathbb{R}^{N}\right)
$$

and

$$
\|u\|_{\infty}=\operatorname{ess} \sup |u(x)|, \quad u \in L^{\infty}\left(\mathbb{R}^{N}\right)
$$

Evidently, $E$ is continuously embedded into $L^{p}\left(\mathbb{R}^{N}\right)$ for $2 \leq p \leq 2^{*}$ under the condition $(\mathrm{V})$, that is, there exists $S_{p}>0$ such that

$$
\|u\|_{p} \leq S_{p}\|u\|, \quad \forall u \in E, p \in\left[2,2^{*}\right]
$$

From [25], we have the following result.

Lemma 2.1 (Bonheure and Mercuri [25]) For any $r>0$, the embedding from $E$ into $L^{p}\left(\bar{B}_{r}\right)$ is compact for $1 \leq p<2^{*}$, where $\bar{B}_{r}=\left\{x \in \mathbb{R}^{N}:|x| \leq r\right\}$ and $2^{*}=2 N /(N-2)$, if $N>2$; $2^{*}=\infty$, if $N=2$.

Just as mentioned in Section 1, the proofs of our results are based on combining Morse theory, a critical groups computation with the minimax method such as the Clark theorem, and local linking. Hence, the following concepts and results are necessary.

Let $X$ be a real Banach space and $J \in C^{1}(E, \mathbb{R})$.

Definition 2.1 (Chang [23]) Let $u$ be an isolated critical point of $J$ with $J(u)=c, c \in \mathbb{R}$, and let $U$ be a neighborhood of $u$, containing the unique critical point. We call

$$
C_{q}(J, u):=H_{q}\left(J^{c} \cap U, J^{c} \cap U \backslash\{u\}\right), \quad q=0,1,2, \ldots,
$$

the $q$ th critical group of $J$ at $u$, where $J^{c}:=\{u \in E: J(u) \leq c\}$ and $H_{q}(\cdot, \cdot)$ stands for the $q$ th singular relative homology group with integer coefficients. 
We say that $u$ is a homological nontrivial critical point of $J$ if at least one of its critical groups is nontrivial.

Proposition 2.1 (Bartsch and $\mathrm{Li}[21]$ ) Let 0 be a critical point of $J$ with $J(0)=0$. Assume that $J$ has a local linking at 0 with respect to $X=W_{1} \oplus W_{2}, k=\operatorname{dim} W_{1}<\infty$, that is, there exists $\rho>0$ small such that

$$
J(u) \leq 0, \quad u \in W_{1},\|u\| \leq \rho \quad \text { and } \quad J(u)>0, \quad u \in W_{2}, 0<\|u\| \leq \rho .
$$

Then $C_{k}(J, 0) \varsubsetneqq 0$, that is, 0 is a homological nontrivial critical point of $J$.

Definition 2.2 We say that $J \in C^{1}(E, \mathbb{R})$ satisfies the (P.S.)-condition if any sequence $\left\{u_{n}\right\}$ in $E$ such that

$$
J\left(u_{n}\right) \rightarrow c, \quad J^{\prime}\left(u_{n}\right) \rightarrow 0, \quad \text { as } n \rightarrow \infty,
$$

has a convergent subsequence.

Proposition 2.2 (Zhang and Li [24]) Assume that J satisfies the (P.S.)-condition and is bounded from below. If $J$ has a critical point that is homological nontrivial and is not the minimizer of $J$, then $J$ has at least three critical points.

Proposition 2.3 (Rabinowitz [26]) Let $X$ be a Banach space, $J \in C^{1}(E, \mathbb{R})$ with $J$ even, bounded from below, and satisfying the (P.S.)-condition. Suppose $J(0)=0$, there is a set $K \subset E$ such that $K$ is homeomorphic to $S^{k-1}$ by an odd map, and $\sup _{K} J(u)<0$. Then $J$ possesses at least $k$ distinct pairs of critical points.

\section{Proofs of main results}

In this section, we are in the position to prove our main results. To this end, we need the following lemmas.

Lemma 3.1 Assume that (V) and (f1) hold. Then the functional $J(u): E \rightarrow \mathbb{R}$ defined by

$$
\begin{aligned}
J(u)= & \frac{1}{2} \int_{\mathbb{R}^{N}}\left[a|\nabla u|^{2}+V(x) u^{2}\right] d x \\
& +\frac{b}{4}\left(\int_{\mathbb{R}^{N}}|\nabla u|^{2} d x\right)^{2}-\int_{\mathbb{R}^{N}} F(x, u) d x
\end{aligned}
$$

is well defined and of class $C^{1}(E, \mathbb{R})$ and

$$
\begin{aligned}
\left\langle J^{\prime}(u), v\right\rangle= & \left(a+b \int_{\mathbb{R}^{N}}|\nabla u|^{2} d x\right) \int_{\mathbb{R}^{N}} \nabla u \nabla v d x \\
& +\int_{\mathbb{R}^{N}} V(x) u v d x-\int_{\mathbb{R}^{N}} f(x, u) v d x, \quad \forall u, v \in E .
\end{aligned}
$$

Furthermore, the critical points of $J(u)$ in $E$ are the solutions of (1.1). 
Proof We divide the proof into three steps.

Step 1. We first show that $J$ is well defined on $E$. It follows from (f1) that

$$
|F(x, u)| \leq \sum_{i=1}^{m} a_{i}(x)|u|^{\delta_{i}}
$$

By (V), (3.3), and the Hölder inequality, for any $u \in E$, we get

$$
\begin{aligned}
\int_{\mathbb{R}^{N}}|F(x, u)| d x & \leq \sum_{i=1}^{m} \int_{\mathbb{R}^{N}} a_{i}(x)|u|^{\delta_{i}} d x \\
& \leq \sum_{i=1}^{m} \int_{\mathbb{R}^{N}}\left(\frac{V(x)}{\alpha}\right)^{\frac{\delta_{i}}{2}} a_{i}(x)|u|^{\delta_{i}} d x \\
& \leq \sum_{i=1}^{m} \alpha^{-\frac{\delta_{i}}{2}}\left\|a_{i}(x)\right\|_{\frac{2}{2-\delta_{i}}}\left(\int_{\mathbb{R}^{N}} V(x)|u|^{2} d x\right)^{\frac{\delta_{i}}{2}} \\
& \leq C_{1} \sum_{i=1}^{m}\|u\|^{\delta_{i}} .
\end{aligned}
$$

Thus, $J(u)$ is well defined on $E$ by (3.1) and (3.4).

Step 2. We are in a position to show that (3.2) holds. By (f1), for any $u, v \in E, l \in(0,1)$, $\theta(x): \mathbb{R}^{N} \rightarrow(0,1)$, and the Hölder inequality, we obtain

$$
\begin{aligned}
& \int_{\mathbb{R}^{N}} \max _{l \in(0,1)}|f(x, u(x)+l \theta(x) v(x)) v(x)| d x \\
& =\int_{\mathbb{R}^{N}} \max _{l \in(0,1)}|f(x, u(x)+l \theta(x) v(x)) \| v(x)| d x \\
& \leq \sum_{i=1}^{m} \delta_{i} \int_{\mathbb{R}^{N}} a_{i}(x)|u(x)+\theta(x) v(x)|^{\delta_{i}-1}|v(x)| d x \\
& \leq C_{2} \sum_{i=1}^{m}\left(\int_{\mathbb{R}^{N}}\left|a_{i}\right|^{\frac{2}{2-\delta_{i}}} d x\right)^{\frac{2-\delta_{i}}{2}}\left(\int_{\mathbb{R}^{N}} V(x)|u(x)|^{2} d x\right)^{\frac{\delta_{i}-1}{2}}\left(\int_{\mathbb{R}^{N}} V(x)|v(x)|^{2} d x\right)^{\frac{1}{2}} \\
& \quad+C_{2} \sum_{i=1}^{m}\left(\int_{\mathbb{R}^{N}}\left|a_{i}\right|^{\frac{2}{2-\delta_{i}}} d x\right)^{\frac{2-\delta_{i}}{2}}\left(\int_{\mathbb{R}^{N}} V(x)|v(x)|^{2} d x\right)^{\frac{\delta_{i}}{2}} \\
& \leq C_{2} \sum_{i=1}^{m}\left\|a_{i}\right\| \frac{2}{2-\delta_{i}}\left(\|u\|^{\delta_{i}-1}+\|v\|^{\delta_{i}-1}\right)\|v\|<+\infty .
\end{aligned}
$$

Then it follows from (3.1), (3.5), and Lebesgue's dominated convergence theorem that

$$
\begin{aligned}
\left\langle J^{\prime}(u), v\right\rangle= & \lim _{l \rightarrow 0^{+}} \frac{J(u+l v)-J(u)}{l} \\
= & {\left[a+\int_{\mathbb{R}^{N}}\left(|\nabla u|^{2}+V(x)|u|^{2}\right) d x\right] \int_{\mathbb{R}^{N}}[\nabla u \nabla v+V(x) u v] d x } \\
& -\lim _{l \rightarrow 0^{+}} \frac{1}{l} \int_{\mathbb{R}^{N}}[F(x, u(x)+l v(x))-F(x, u(x))] d x \\
= & {\left[a+\int_{\mathbb{R}^{N}}\left(|\nabla u|^{2}+V(x)|u|^{2}\right) d x\right] \int_{\mathbb{R}^{N}}[\nabla u \nabla v+V(x) u v] d x }
\end{aligned}
$$




$$
\begin{aligned}
& -\lim _{l \rightarrow 0^{+}} \int_{\mathbb{R}^{N}} f(x, u(x)+\theta(x) l v(x)) v(x) d x \\
= & {\left[a+\int_{\mathbb{R}^{N}}\left(|\nabla u|^{2}+V(x)|u|^{2}\right) d x\right] \int_{\mathbb{R}^{N}}[\nabla u \nabla v+V(x) u v] d x } \\
& -\int_{\mathbb{R}^{N}} f(x, u(x)) v(x) d x
\end{aligned}
$$

which implies that (3.2) holds. Moreover, by a standard argument, it is easy to show that the critical points of $J(u)$ in $E$ are the solutions of problem (1.1) (see for example [27]).

Step 3. We finally show that $J^{\prime}(u)$ is continuous. According to (3.1), it suffices to show that

$$
\Phi^{\prime} \triangleq \int_{\mathbb{R}^{N}} f(x, u) v d x
$$

is continuous. Let $u_{k} \rightarrow u$ in $E$. Then

$$
u_{k} \rightarrow u, \quad \text { in } L^{2}\left(\mathbb{R}^{N}\right), \quad u_{k} \rightarrow u \quad \text { a.e. in } \mathbb{R}^{N}
$$

We shall show that

$$
\int_{\mathbb{R}^{N}}\left|f\left(x, u_{k}\right)-f(x, u)\right|^{2} d x \rightarrow 0, \quad \text { as } k \rightarrow+\infty .
$$

To this end, arguing by contradiction, suppose that there exist a constant $\varepsilon_{0}>0$ and a subsequence (also denoted $\left\{u_{k}\right\}$ ) such that

$$
\int_{\mathbb{R}^{N}}\left|f\left(x, u_{k}\right)-f(x, u)\right|^{2} d x \geq \varepsilon_{0}, \quad \text { as } k \rightarrow+\infty .
$$

Since $u_{k} \rightarrow u$ in $L^{2}\left(\mathbb{R}^{N}\right)$, passing to a subsequence if necessary we can assume that $\sum_{i=1}^{\infty}\left\|u_{k}-u\right\|_{2}^{2}<+\infty$. Set $w(x)=\left[\sum_{i=1}^{\infty}\left|u_{k}-u\right|^{2}\right]^{\frac{1}{2}}, x \in \mathbb{R}^{N}$. Then $w(x) \in L^{2}\left(\mathbb{R}^{N}\right)$. For all $k \in \mathbb{N}$ and by (f1), we have

$$
\begin{aligned}
\left|f\left(x, u_{k}(x)\right)-f(x, u(x))\right|^{2} & \leq 2\left|f\left(x, u_{k}(x)\right)\right|^{2}+2|f(x, u(x))|^{2} \\
& \leq C_{3} \sum_{i=1}^{m}\left|a_{i}(x)\right|^{2}\left[\left|u_{k}(x)\right|^{2 \delta_{i}-2}+|u(x)|^{2 \delta_{i}-2}\right] \\
& \leq C_{3} \sum_{i=1}^{m}\left|a_{i}(x)\right|^{2}\left[|w(x)|^{2 \delta_{i}-2}+|u(x)|^{2 \delta_{i}-2}\right] \\
& :=g(x), \quad \text { a.e. in } \mathbb{R}^{N}
\end{aligned}
$$

and

$$
\begin{aligned}
\int_{\mathbb{R}^{N}} g(x) d x & =C_{3} \sum_{i=1}^{m} \int_{\mathbb{R}^{N}}\left|a_{i}(x)\right|^{2}\left[|w(x)|^{2 \delta_{i}-2}+|u(x)|^{2 \delta_{i}-2}\right] d x \\
& \leq C_{3} \sum_{i=1}^{m}\left\|a_{i}(x)\right\|_{\frac{2}{2-\delta_{i}}}^{2}\left[\|w(x)\|_{2}^{2 \delta_{i}-2}+\|u(x)\|_{2}^{2 \delta_{i}-2}\right]<+\infty .
\end{aligned}
$$


Then combining (3.7), (3.10), (3.11) with Lebesgue's dominated convergence theorem yields

$$
\int_{\mathbb{R}^{N}}\left|f\left(x, u_{k}\right)-f(x, u)\right|^{2} d x \rightarrow 0, \quad \text { as } k \rightarrow+\infty,
$$

which contradicts (3.9). Thus, (3.8) holds.

For all given $v \in E$, one deduces from (3.6), (3.12), and the Hölder inequality that

$$
\begin{aligned}
\left|\left\langle\Phi^{\prime}\left(u_{k}\right)-\Phi^{\prime}(u), v\right\rangle\right| & =\left|\int_{\mathbb{R}^{N}}\left[f\left(x, u_{k}\right)-f(x, u)\right] v d x\right| \\
& \leq C_{4}\|v\|\left(\int_{\mathbb{R}^{N}}\left|f\left(x, u_{k}\right)-f(x, u)\right|^{2} d x\right)^{\frac{1}{2}} \rightarrow 0,
\end{aligned}
$$

as $k \rightarrow+\infty$, implying the continuity of $\Phi^{\prime}$. Hence, $J \in C^{1}(E, \mathbb{R})$. The proof is completed.

Lemma 3.2 Assume that the conditions (V) and (f1) hold, then $J(u)$ is bounded from below and satisfies the (P.S.)-condition.

Proof Firstly, we show that $J$ is bounded from below. By (3.1), (f1), and the Hölder inequality, we have

$$
\begin{aligned}
J(u) & \geq \frac{1}{2} \min \{a, 1\}\|u\|^{2}-\int_{\mathbb{R}^{N}} F(x, u) d x \\
& \geq \frac{1}{2} \min \{a, 1\}\|u\|^{2}-\sum_{i=1}^{m} \int_{\mathbb{R}^{N}} a_{i}(x)|u|^{\delta_{i}} d x \\
& \geq \frac{1}{2} \min \{a, 1\}\|u\|^{2}-C_{5} \sum_{i=1}^{m}\left(\int_{\mathbb{R}^{N}}\left|a_{i}(x)\right|^{\frac{2}{2-\delta_{i}}} d x\right)^{\frac{2-\delta_{i}}{2}}\left(\int_{\mathbb{R}^{N}} V(x)|u|^{2} d x\right)^{\frac{\delta_{i}}{2}} \\
& \geq \frac{1}{2} \min \{a, 1\}\|u\|^{2}-C_{5} \sum_{i=1}^{m}\left\|a_{i}\right\|_{\frac{2}{2-\delta_{i}}}\|u\|^{\delta_{i}} .
\end{aligned}
$$

Since $1<\delta_{i}<2(i=1,2, \ldots, m)$, (3.13) implies that $J(u) \rightarrow+\infty$ as $\|u\| \rightarrow+\infty$. Consequently, $J$ is bounded from below.

Next, we prove that $J$ satisfies the (P.S.)-condition. Assume that $\left\{u_{n}\right\} \subset E$ is a sequence such that (2.2) holds. Then by (3.13), there exists a constant $M>0$ such that

$$
\left\|u_{n}\right\| \leq M, \quad \forall n \in \mathbb{N}
$$

Going if necessary to a subsequence we can assume that $u_{n} \rightarrow u_{0}$ in $E$, then $\left\|u_{0}\right\| \leq M$. Hence, using Lemma 2.1 implies that

$$
u_{n} \rightarrow u_{0}, \quad \text { in } L_{\mathrm{loc}}^{s}\left(\mathbb{R}^{N}\right), s \in\left[2,2^{*}\right) .
$$

By (f1), for any given $\varepsilon>0$, we can choose $\rho>0$ such that

$$
\left(\int_{|x| \geq \rho}\left|a_{i}\right|^{\frac{2}{2-\delta_{i}}} d x\right)^{\frac{2-\delta_{i}}{2}}<\varepsilon .
$$


It follows from (f1), (3.14), (3.15), and the Hölder inequality that there exists $n_{0} \in \mathbb{N}$ such that

$$
\begin{aligned}
& \int_{|x| \leq \rho}\left|f\left(x, u_{n}\right)-f\left(x, u_{0}\right)\right|\left|u_{n}-u_{0}\right| d x \\
& \quad \leq\left(\int_{|x| \leq \rho}\left|f\left(x, u_{n}\right)-f\left(x, u_{0}\right)\right|^{2} d x\right)^{\frac{1}{2}}\left(\int_{|x| \leq \rho}\left|u_{n}-u_{0}\right|^{2} d x\right)^{\frac{1}{2}} \\
& \quad \leq \varepsilon\left(\int_{|x| \leq \rho}\left|f\left(x, u_{n}\right)-f\left(x, u_{0}\right)\right|^{2} d x\right)^{\frac{1}{2}} \\
& \quad \leq C_{6} \varepsilon\left[\sum_{i=1}^{m}\left(\left\|u_{n}\right\|_{2}^{2 \delta_{i}-2}+\left\|u_{0}\right\|_{2}^{2 \delta_{i}-2}\right)\right]^{\frac{1}{2}} \\
& \quad \leq C_{7} \varepsilon
\end{aligned}
$$

for $n \geq n_{0}$.

On the other hand, combining (2.1), (3.16) with the Hölder inequality yields

$$
\begin{aligned}
& \int_{|x|>\rho}\left|f\left(x, u_{n}\right)-f\left(x, u_{0}\right)\right|\left|u_{n}-u_{0}\right| d x \\
& \quad \leq \int_{|x|>\rho}\left[\left|f\left(x, u_{n}\right)\right|+\left|f\left(x, u_{0}\right)\right|\right]\left(\left|u_{n}-u_{0}\right|\right) d x \\
& \quad \leq \sum_{i=1}^{m} \delta_{i} \int_{|x|>\rho}\left|a_{i}(x)\right|\left[\left|u_{n}\right|^{\delta_{i}}+\left|u_{0}\right|^{\delta_{i}}+\left|u_{n}\right|^{\delta_{i}-1}\left|u_{0}\right|+\left|u_{0}\right|^{\delta_{i}-1}\left|u_{n}\right|\right] d x \\
& \quad \leq \varepsilon \sum_{i=1}^{m} \delta_{i}\left(\left\|u_{n}\right\|_{2}^{\delta_{i}}+\left\|u_{0}\right\|_{2}^{\delta_{i}}+\left\|u_{n}\right\|_{2}^{\delta_{i}-1}\left\|u_{0}\right\|_{2}+\left\|u_{0}\right\|_{2}^{\delta_{i}-1}\left\|u_{n}\right\|_{2}\right) \\
& \leq C_{8} \varepsilon .
\end{aligned}
$$

Since $\varepsilon$ is arbitrary, (3.17) and (3.18) give

$$
\int_{\mathbb{R}^{N}}\left|f\left(x, u_{n}\right)-f\left(x, u_{0}\right)\right|\left|u_{n}-u_{0}\right| d x \rightarrow 0, \quad \text { as } n \rightarrow+\infty .
$$

From (3.2) we have

$$
\begin{aligned}
\min & \{a, 1\}\left\|u_{n}-u_{0}\right\|^{2} \\
\leq & \left\langle J^{\prime}\left(u_{n}\right)-J^{\prime}\left(u_{0}\right), u_{n}-u_{0}\right\rangle \\
& +b\left(\int_{\mathbb{R}^{N}}|\nabla u|^{2} d x-\int_{\mathbb{R}^{N}}\left|\nabla u_{n}\right|^{2} d x\right) \int_{\mathbb{R}^{N}} \nabla u \nabla\left(u_{n}-u\right) d x \\
& +\int_{\mathbb{R}^{N}}\left[f\left(x, u_{n}\right)-f\left(x, u_{0}\right)\right]\left(u_{n}-u_{0}\right) d x .
\end{aligned}
$$

Obviously,

$$
\left\langle J^{\prime}\left(u_{n}\right)-J^{\prime}\left(u_{0}\right), u_{n}-u_{0}\right\rangle \rightarrow 0, \quad \text { as } n \rightarrow+\infty .
$$


By Lemma 2 in [5], we have

$$
b\left(\int_{\mathbb{R}^{N}}|\nabla u|^{2} d x-\int_{\mathbb{R}^{N}}\left|\nabla u_{n}\right|^{2} d x\right) \int_{\mathbb{R}^{N}} \nabla u \nabla\left(u_{n}-u\right) d x \rightarrow 0, \quad \text { as } n \rightarrow+\infty .
$$

In view of (3.19), (3.20), (3.21), and (3.22), we see that $u_{n} \rightarrow u_{0}$ in $E$. Hence, $J$ satisfies the (P.S.)-condition. The proof is completed.

Now, we choose an orthogonal basis $\left\{e_{j}\right\}$ of $E$ and let

$$
X_{j}:=\operatorname{span}\left\{e_{1}, \ldots, e_{j}\right\} \quad \text { and } \quad Y_{j}:=X_{j}^{\perp} .
$$

Then $E=X_{j} \oplus Y_{j}$.

Lemma 3.3 Assume that assumptions (V), (f1), and (f2) are satisfied. Then $C_{k}(J, 0) \varsubsetneqq 0$.

Proof Let $W_{1}=X_{k}, W_{2}=Y_{k}$. Then $\operatorname{dim} W_{1}=k<\infty$. Obviously, $J(0)=0$. Now, we show that the functional $J$ has a local linking at 0 .

On the one hand, for any $u \in W_{1}$, utilizing (f2) gives

$$
\begin{aligned}
J(u) & \leq \frac{1}{2} \max \{a, 1\}\|u\|^{2}+\frac{b}{4}\|u\|^{4}-\int_{\mathbb{R}^{N}} F(x, u) d x \\
& \leq \frac{1}{2} \max \{a, 1\}\|u\|^{2}+\frac{b}{4}\|u\|^{4}-c_{1}\|u\|_{\kappa}^{\kappa} .
\end{aligned}
$$

Since all norms on a finite dimensional space are equivalent, we deduce from (3.24) that

$$
J(u) \leq \frac{1}{2} \max \{a, 1\}\|u\|^{2}+\frac{b}{4}\|u\|^{4}-C_{9}\|u\|^{\kappa} .
$$

Therefore, from the above inequality, we have $J(u) \leq 0=J(0)$ if we take $\|u\|=\rho$ small for all $u \in W_{1}$ since $\kappa \in(1,2)$.

On the other hand, for $u \in W_{2}$, combining (2.1) and (f2) yields

$$
\begin{aligned}
J(u) & \geq \frac{1}{2} \min \{a, 1\}\|u\|^{2}-\int_{\mathbb{R}^{N}} F(x, u) d x \\
& \geq \frac{1}{2} \min \{a, 1\}\|u\|^{2}-c_{2} \int_{\mathbb{R}^{N}}|u|^{\vartheta} d x \\
& \geq \frac{1}{2} \min \{a, 1\}\|u\|^{2}-C_{10}\|u\|^{\vartheta} .
\end{aligned}
$$

Hence, we can derive that $J(u)>0$ if $u \in W_{2}$ with $\|u\|=\rho$ small since $2<\vartheta \leq 2^{*}$.

Therefore, combining the above arguments with Proposition 2.1, we have $C_{k}(J, 0) \supsetneqq 0$. This means that 0 is a homological nontrivial critical point of $J$. We complete the proof.

In what follows, we give the proofs of Theorem 1.1 and Theorem 1.2.

Proof of Theorem 1.1 In view of Lemma 3.1, $J \in C^{1}(E, \mathbb{R})$. By Lemma 3.2, $J$ is bounded from below and satisfies the (P.S.)-condition. Lemma 3.3 shows that $0 \in E$ is a homologically nontrivial critical point of $J$ but not a minimizer. Then the conclusion follows from Proposition 2.2. 
Proof of Theorem 1.2 Lemma 3.1 shows that $J \in C^{1}(E, \mathbb{R})$. By (f3) and (3.1), we can easily check that functional $J$ is even and satisfies $J(0)=0$. It follows from Lemma 3.2 that $J$ satisfies the (P.S.)-condition and is bounded from below in $E$.

For $\rho>0$, let $K=B_{\rho}=\left\{u \in X_{k}:\|u\|=\rho\right\} \subset E$. Obviously, there is an identity operator such that $K$ is homeomorphic to $S^{k-1}$. From (f2), we have

$$
\begin{aligned}
\sup _{B_{\rho}} J(u) & \leq \frac{1}{2} \max \{a, 1\}\|u\|^{2}+\frac{b}{4}\|u\|^{4}-\int_{\mathbb{R}^{N}} F(x, u) d x \\
& \leq \frac{1}{2} \max \{a, 1\}\|u\|^{2}+\frac{b}{4}\|u\|^{4}-c_{1}\|u\|_{\kappa}^{\kappa}, \quad u \in B_{\rho} .
\end{aligned}
$$

Since all norms are equivalent on a finite dimensional space, it follows from the above inequality that

$$
\sup _{B_{\rho}} J(u) \leq \frac{1}{2} \max \{a, 1\}\|u\|^{2}+\frac{b}{4}\|u\|^{4}-C_{11}\|u\|^{\kappa}, \quad u \in B_{\rho},
$$

which implies that $\sup _{K} J(u)<0$ if $\rho>0$ is sufficiently small.

By the definition of $X_{k}$, we have $\operatorname{dim} X_{k}=k<\infty$. Thus, all the conditions of Proposition 2.3 are satisfied. Therefore, $J$ has at least $k$ distinct pairs of critical points. This completes the proof.

\section{Competing interests}

The authors declare that they have no competing interests.

\section{Authors' contributions}

All authors contributed equally to the writing of this paper. All authors read and approved the final manuscript.

\section{Author details}

${ }^{1}$ School of Mathematics and Statistics, Central South University, Changsha, Hunan 410083, P.R. China. ${ }^{2}$ School of Mathematics and Computer Sciences, Yichun University, Yichun, 336000, P.R. China.

\section{Acknowledgements}

The first author was partially supported by Hunan Provincial Foundation For Postgraduate (Grant No. CX2014B044). The second author was partially supported by Natural Science Foundation of China (Grant No. 11271372) and Mathematics and Interdisciplinary Sciences project of CSU. The third author was partially supported by Natural Science Foundation of China (Grant No. 11561068).

Received: 24 August 2015 Accepted: 6 October 2015 Published online: 14 October 2015

\section{References}

1. Kirchhoff, G: Mechanik. Teubner, Leipzig (1883)

2. Chipot, M, Lovat, B: Some remarks on nonlocal elliptic and parabolic problems. Nonlinear Anal. 30, 4619-4627 (1997)

3. Corrêa, F: On positive solutions of nonlocal and nonvariational elliptic problems. Nonlinear Anal. 59, 1147-1155 (2004)

4. Lions, J: On some questions in boundary value problems of mathematical physics. In: Contemporary Developments in Continuum Mechanics and Partial Differential Equations. Proc. Internat. Sympos., Inst. Mat., Univ. Fed. Rio de Janeiro, Rio de Janeiro, 1977. North-Holland Math. Stud., vol. 30, pp. 284-346. North-Holland, Amsterdam (1978)

5. Wu, X: Existence of nontrivial solutions and high energy solutions for Schrödinger-Kirchhoff-type equations in $\mathbb{R}^{N}$. Nonlinear Anal., Real World Appl. 12, 1278-1287 (2011)

6. Sun, J, Wu, T: Ground state solutions for an indefinite Kirchhoff type problem with steep potential well. J. Differ. Equ. 256, 1771-1792 (2014)

7. Liu, W, He, X: Multiplicity of high energy solutions for superlinear Kirchhoff equations. J. Appl. Math. Comput. 39, 473-487 (2012)

8. He, X, Zou, W: Existence and concentration behavior of positive solutions for a Kirchhoff equations in $\mathbb{R}^{3}$. J. Differ. Equ. 252, 1813-1834 (2012)

9. Alves, C, Corrèa, F, Ma, T: Positive solutions for a quasilinear elliptic equation of Kirchhoff type. Comput. Math. Appl. 49, 85-93 (2005)

10. Cheng, B, Wu, X: Existence results of positive solutions of Kirchhoff type problems. Nonlinear Anal. 71, 4883-4892 (2009) 
11. He, X, Zou, W: Infinitely many positive solutions for Kirchhoff-type problems. Nonlinear Anal. 70, 1407-1414 (2009)

12. Li, Y, Li, F, Shi, J: Existence of a positive solution to Kirchhoff type problems without compactness conditions. J. Differ. Equ. 253, 2285-2294 (2012)

13. Liu, Z, Guo, S: Existence and concentration of positive ground states for a Kirchhoff equation involving critical Sobolev exponent. Z. Angew. Math. Phys. 66, 747-769 (2015)

14. Liu, Z, Guo, S: Existence of positive ground state solutions for Kirchhoff type problems. Nonlinear Anal. 120, 1-13 (2015)

15. Liu, Z, Guo, S: On ground states for the Kirchhoff-type problem with a general critical nonlinearity. J. Math. Anal. Appl. 426, 267-287 (2015)

16. Mao, A, Zhang, Z: Sign-changing and multiple solutions of Kirchhoff type problems without P.S. condition. Nonlinear Anal. 70, 1275-1287 (2009)

17. Perera, K, Zhang, Z: Nontrivial solutions of Kirchhoff-type problems via the Yang index. J. Differ. Equ. 221, 246-255 (2006)

18. $\mathrm{Xu}, \mathrm{L}, \mathrm{Chen}, \mathrm{H}$ : Existence and multiplicity of solutions for fourth-order elliptic equations of Kirchhoff type via genus theory. Bound. Value Probl. 2014, 212 (2014)

19. Wu, X: High energy solutions of systems of Kirchhoff-type equations in $\mathbb{R}^{N}$. J. Math. Phys. 53, 1-18 (2012)

20. Duan, L, Huang, L: Infinitely many solutions for sublinear Schrödinger-Kirchhoff-type equations with general potentials. Results Math. 66, 181-197 (2014)

21. Bartsch, $T, L i, S$ : Critical point theory for asymptotically quadratic functionals and applications to problems with resonance. Nonlinear Anal. 28, 419-441 (1997)

22. Liu, J: The Morse index of a saddle point. Syst. Sci. Math. Sci. 2, 32-39 (1989)

23. Chang, K: Infinite Dimensional Morse Theory and Multiple Solution Problems. Birkhäuser, Boston (1993)

24. Zhang, J, Li, S: Multiple nontrivial solutions for some fourth-order semilinear elliptic problems. Nonlinear Anal. 60 , 221-230 (2005)

25. Bonheure, D, Mercuri, C: Embedding theorems and existence results for nonlinear Schrödinger-Poisson systems with unbounded and vanishing potentials. J. Differ. Equ. 251, 1056-1085 (2011)

26. Rabinowitz, P: Minimax Methods in Critical Point Theory with Applications to Differential Equations. CBMS Reg. Conf. Ser. in Math., vol. 65. Am. Math. Soc., Providence (1986)

27. Benci, V, Fortunato, D: An eigenvalue problem for the Schrödinger-Maxwell equations. Topol. Methods Nonlinear Anal. 11, 283-293 (1998)

\section{Submit your manuscript to a SpringerOpen ${ }^{\circ}$ journal and benefit from:}

- Convenient online submission

- Rigorous peer review

- Immediate publication on acceptance

- Open access: articles freely available online

- High visibility within the field

- Retaining the copyright to your article 